\title{
Electronic structure, magnetic properties, and microstructural analysis of thiol-functionalized Au nanoparticles: Role of chemical and structural parameters in the ferromagnetic behaviour
}

\author{
Estefanía Guerrero · Miguel A. Muñoz-Márquez . \\ Enrique Fernández-Pinel · Patricia Crespo · \\ Antonio Hernando · Asunción Fernández
}

Received: date / Accepted: date

\begin{abstract}
Gold nanoparticles (NPs) have been stabilized with a variety of thiol-containing molecules in order to change their chemical and physical properties; among the possible capping systems, alkane chains with different lengths, a carboxylic acid and a thiol-containing biomolecule (tiopronin) have been selected as protecting shells for the synthesized NPs; the NPs solubility in water or organic solvents is determined by the protecting molecule. A full microstructural characterization of these NPs is presented in the current research work. It has been shown that NPs capped with alkanethiol chains have a marked ferromagnetic behaviour which might be also dependent on the chain length. The simultaneous presence of $\mathrm{Au}-\mathrm{Au}$ and $\mathrm{Au}-\mathrm{S}$ bonds together with a reduced particle diameter, and the formation of an ordered monolayer protective shell, have been proved to be key parameters for the ferromagnetic-like behaviour exhibited by thiol-functionalized gold NPs.
\end{abstract}

Keywords gold nanoparticles $\cdot$ microstructure $\cdot$ ferromagnetic behaviour

\section{Introduction}

Transition metal nanoparticles (hereafter NPs) are one of the most-studied systems due to their novel electronic, optical and magnetic properties. In addition, these nanostructured systems play an important role in many different fields of science such as chemical catalysis, nanoelectronics, biology and, recently, in biomedical applications, for instance, the labeling

E. Guerrero · M. A. Muñoz-Márquez (corresponding author) · A. Fernández

Instituto de Ciencia de Materiales de Sevilla (CSIC-US), Av. Americo Vespucio 49, 41092 - Sevilla, Spain Tel.: +34-954-489500 ext. 9218

Fax: +34-954-460665

E-mail: miguel.angel@icmse.csic.es

E. Fernández-Pinel · P. Crespo · A. Hernando

Instituto de Magnetismo Aplicado (UCM-ADIF-CSIC), P.O. Box 155, 28230 - Las Rozas, Madrid, Spain

P. Crespo $\cdot$ A. Hernando

Departamento de Física de Materiales, UCM, Av. Complutense s/n, 28040 - Madrid, Spain 
of targeting cells with nanoparticles as reported by de la Fuente et al (2006). Particularly, the magnetic properties of clusters of $4 d$ elements, which are non-magnetic as bulk materials, have attracted much of the attention (e.g. Kumar and Kawazoe, 2003; Shinohara et al, 2003; Sampedro et al, 2003; Litrán et al, 2006; Hernando et al, 2006c); among these $4 d$ transition metals, gold has been the target of many investigations either as thin films with or without an organic layer on top (Carmeli et al, 2003; Reich et al, 2006), or as nanometric particles with our without capping molecules (Hori et al, 1999; Crespo et al, 2004). Despite all the efforts in this field, the permanent magnetism shown by these systems is not yet understood (Vager and Naaman, 2004; Hernando and García, 2006; Hernando et al, 2006a; Crespo et al, 2006). On this matter, studies regarding the origins of the ferromagnetic-like behaviour (Yamamoto et al, 2004; Negishi et al, 2006) were performed by means of x-ray magnetic circular dichroism (XMCD); unfortunately, in spite of having two research works trying to discern the origins of such a particular magnetic behaviour, the reason behind it still remains unclear. Recently, a set of new experiments was performed in our research group that studied the influence of the particle diameter in the magnetic behaviour of thiol-capped gold NPs (Guerrero et al, 2007). Even though there has been a wealth of experimental work in these systems, there is a need of investigating new structural possibilities such as capping molecules, functional groups, particle morphology and more about electronic and magnetic properties in order to systematically increase the knowledge of these nanostructured systems.

Based on the method proposed by Brust et al (1994) to synthesize alkanethiolate-capped gold NPs, we have produced gold clusters functionalized through $\mathrm{Au}-\mathrm{S}$ bonds, with dodecanethiol and octanethiol molecules, samples named $\mathrm{Au}-\mathrm{SC}_{12}$ and $\mathrm{Au}-\mathrm{SC}_{8}$ respectively. Simard et al (2000) developed a synthesis method that we have used to produce gold NPs simultaneously capped with both octanethiol and thiolated undecanoic acid molecules (sample labelled $\mathrm{Au}-\mathrm{SC}_{8} / \mathrm{SC}_{11} \mathrm{COOH}$ ). Finally, following the synthesis method presented by Templeton et al (1999), we have synthesized water soluble gold NPs capped with tiopronin (Au-ST), which is a synthetic thiol-containing biomolecule.

In all the synthesized samples, we have carried out a thorough study of the microstructure, chemical composition and magnetic properties, in order to understand the nature of the nanostructures which in some cases are responsible of the singular ferromagnetic behaviour. Furthermore, the solubility properties in organic and aqueous solvents along with the functionalization possibilities that offer the used thiol-containing protective shells, open a wide range of options, specially in biomedical applications.

\section{Theoretical background}

As described in Sect. 1, combined theory-experiment papers have recently appeared which report a new theoretical explanation and compare such theory with somewhat newer superconducting quantum interference device (SQUID) magnetometry results (Vager and Naaman, 2004; Hernando and García, 2006; Hernando et al, 2006a). These papers claim to offer a more than plausible explanation that would reconcile theory and experiment; the high magnetic anisotropy required to block up the magnetic moment of Au NPs, with a diameter lower than $2 \mathrm{~nm}$, up to room temperature suggests that orbital contribution to the magnetic moment can not be disregarded. The theory proposed by Hernando et al (2006a), which reasonably explains the orbital magnetism observed in different nanostructured films, assumes the induction of orbital motion of surface electrons around ordered arrays of Au-S bonds. It is considered that electrons are pumped up from the substrate to the molecular layer (Vager 
and Naaman, 2004), at the same time, spin-orbit effects, known to be extremely important in gold surfaces, must be taken into account: a strong spin-orbit coupling $\left(\alpha_{r} \hbar^{2}=0.4 \mathrm{eV}\right.$, where $\alpha_{r}$ is the splitting strength of the $p$ band) has been measured by LaShell et al (1996) for gold surfaces by means of angle resolved photoemission spectroscopy and, theoretically described within a nearly-free-electron model by Petersen and Hedegard (2000). Based on the latter statements, Hernando et al (2006a) proposed that the $L_{z}$ dependent part of the Hamiltonian $H$, where $z$ stands for the direction normal to the surface plane, can be written as

$$
H=\frac{L_{z}^{2} \hbar 2}{2 m \xi^{2}}-\alpha_{r} L_{z} s_{z} \hbar^{2}
$$

here $\xi$ is the radius of the thiol-capped region and, $m$ and $s_{z}$ are the mass and the spin component along the $z$ axis of the free electron respectively. The value of the quantum number $L_{z}$ that minimizes Eq. 1 is given by

$$
L_{z}=m \xi^{2} \alpha_{r} s_{z}
$$

This spin-orbit interaction together with a large radius of the self-assembled thiolated molecule domains should be the driving force for inducting orbital motion as claimed by Hernando et al (2006a,b).

Despite the small gold clusters structure remains controversial in the range from four to 30 atom clusters (e.g. Koskinen et al, 2006; Iwasa and Nobusada, 2007) -please note that the proposed models have different structures depending on the number of atoms involved in the cluster- it seems to be widely accepted that in the range 2-10 nm, gold NPs are most likely faceted clusters with a close-packed structure (e.g. Chushak and Bartell, 2000; Yacamán et al, 2001; López-Cartes et al, 2005). In addition, spontaneous reconstruction of (001) gold nanofilms into (111) close-packed structures has been previously reported by Kondo et al (1999); this profound atomic reorganization has been studied by Takayanagi's group in the past which has observed atomic movements in gold nanofilms (e.g. Kondo and Takayanagi, 1997) and in gold fine particles (e.g. Mitome et al, 1990) with diameters below $10 \mathrm{~nm}$. If a protecting molecule is included in the system, the molecule-substrate interface, which strongly influences the molecular ordering, will still remain a controversial point. On this matter, the thiol-induced reconstructions of gold (111) surfaces has been subject of study by Yu et al (2006): the thiol adsorption induces a strong modification of the $\mathrm{Au}$ atomic position in the outermost layer that leads to a variety of surface reconstructions depending on the adsorbed thiolated molecule, which is in agreement with the ability exhibited by thiol-derivatized gold NPs to exchange ligands as proved by various research groups, mentioned throughout the current manuscript (specially in Sect. 3.1), dedicated to study the nanoparticle synthesis process. Moreover, all these research works indicate that the surface rigidity, i.e. surface atoms mobility, is similar in both two-dimensional systems and nanoparticle facets. Therefore, the theory developed for two-dimensional systems by Hernando et al (2006a) could be extrapolated to Au NPs. It is to be noted that at least a fraction of the surface electrons have to keep their mobility or delocalization in order to be available for being captured in the orbits. In any case, regardless of what the details about the microscopic origins of the magnetization are, it seems to be well established that the magnetization arises from modifications of the NPs surface electronic structure biased by the binding between Au atoms and the organic molecules. 


\section{Experimental details}

3.1 Sample preparation

The alkanethiolate-capped gold NPs have been obtained from a liquid-liquid phase reduction at room temperature following the method proposed by Brust et al (1994); the Au:thiol molar ratio was set to $1: 2$, dodecanethiol and octanethiol were used to synthesize two different samples: one with long alkane chains $\left(\mathrm{Au}-\mathrm{SC}_{12}\right)$ and another one with shorter alkane chains $\left(\mathrm{Au}-\mathrm{SC}_{8}\right.$ ). First, $\mathrm{Au}(\mathrm{III})$ is transferred from an aqueous solution containing $\mathrm{HAuCl}_{4}$ (Fluka $99 \%, 0.064 \mathrm{~g}$ in $6.4 \mathrm{ml}$ of milli-Q water) to degassed and dried toluene: tetraoctylammonium bromide (Aldrich $98 \%, 0.112 \mathrm{~g}$ in $20 \mathrm{ml}$ of toluene) is used as the phase-transfer agent. The mixture is strongly stirred for 10 minutes. Once the aqueous phase is removed, $0.1 \mathrm{ml}$ of dodecanethiol (for the $\mathrm{Au}-\mathrm{SC}_{12}$ sample) or $0.07 \mathrm{ml}$ of octanethiol (for the $\mathrm{Au}-\mathrm{SC}_{8}$ sample) are added to the organic phase under strong stirring for 5 minutes; both thiols were purchased from Aldrich and are $98.5 \%$ pure. Then, the formed Au-SR polymeric precursors $\left(\mathrm{R}=\mathrm{C}_{12}\right.$ or $\mathrm{R}=\mathrm{C}_{8}$ ) are reduced with an aqueous solution of $\mathrm{NaBH}_{4}$ (Aldrich $99 \%, 0.1 \mathrm{~g}$ in $6 \mathrm{ml}$ of milli$\mathrm{Q}$ water) which is a reducing agent. The presence of an alkanethiol, either dodecanethiol or octanethiol, leads to the formation of Au-S bonds which isolate the metal clusters preventing them from agglomeration. Subsequently, the organic phase was decanted from the aqueous phase and the toluene was removed under low pressure by means of a rotary evaporator. Finally, the resulting Au NPs were precipitated with ethanol, filtered, washed and dried.

A new type of particles capped with two different ligands at the same time were synthesized by the method proposed by Simard et al (2000). To $60 \mathrm{mg}$ of Au-SC 8 NPs we added $317.1 \mathrm{mg}$ of 11-thioundecanoic acid (Aldrich $98 \%$ ) diluted in $2 \mathrm{ml}$ of tetrahydrofuran. This mixture was stirred in nitrogen atmosphere during two days. Then, the organic solvent was removed under low pressure by means of a rotary evaporator. Finally, the solid phase was filtered and washed with dichloromethane; this sample was labeled as $\mathrm{Au}-\mathrm{SC}_{8} / \mathrm{SC}_{11} \mathrm{COOH}$.

The last system to be synthesized was a set of $\mathrm{Au}$ NPs capped with tiopronin, which is a synthetic thiol-containing biomolecule $\left(\mathrm{C}_{5} \mathrm{H}_{9} \mathrm{NO}_{3} \mathrm{~S}\right)$. In this case, the followed method was the one reported by Templeton et al (1999). The process starts by adding $0.060 \mathrm{~g}$ of

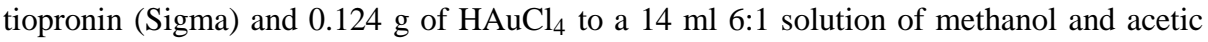
acid. Once this has been done, the solution turns into a red liquid. Then $0.24 \mathrm{~g}$ of reducing agent $\left(\mathrm{NaBH}_{4}\right.$ dissolved in $6 \mathrm{ml}$ of milli-Q water) are added to the previous solution. The mixture is quickly stirred and turns immediately into a black suspension which will be stirred for 45 minutes. The next step is the solvent removal in low pressure by means of a rotary evaporator. Now, $25 \mathrm{ml}$ of milli-Q water are added to the system and the acidity is corrected to $\mathrm{pH}=1$ with concentrated $\mathrm{HCl}$. The solution is then purified by dialyzis using cellulose membranes (Spectra/Por CE, MWCO=10000). The dialyzis process lasted 72 hours and milli-Q water was refilled every ten hours. Finally, water is removed by lyophilization.

\subsection{Sample characterization}

The iron and gold content in the studied samples was evaluated by inductively coupled plasma-atomic emission spectrometry analysis (ICP). The amount of light elements in the samples such as carbon, hydrogen, nitrogen and sulphur, was determined by elemental chemical analysis. The chemical composition of the samples was also measured by energy dispersive X-ray analysis (EDX) in a transmission electron microscope (TEM). 
TEM analysis was carried out in a Philips CM200 microscope working at $200 \mathrm{kV}$. The approximate particle size distribution histograms were measured using an image analyzer software that determines the cluster radii from a digital image of the micrographs.

X-ray absorption spectra (XAS) of the Au NPs were recorded in transmission mode at the BM29 beamline of ESRF. The samples were measured as thin self-supported pressed pellets. Spectra were recorded at the $\mathrm{Au} \mathrm{L}_{2}$ - and $\mathrm{L}_{3}$-edge, at 13734 and $11919 \mathrm{eV}$ respectively. The extended X-ray absorption fine structure (EXAFS) oscillations were quantitatively analyzed with the software package developed by Bonin et al (1989). The coordination numbers $(\mathrm{N})$, bond distances $(\mathrm{R})$ and Debye-Waller factors $(\sigma)$ were extracted using a least-square fitting procedure that uses the theoretical phases and amplitudes proposed by Zabinsky et al (1995). These parameters have been previously compared to $\mathrm{Au}$ foil and $\mathrm{Au}_{2} \mathrm{~S}$ (Aldrich, 99.9\%) reference samples.

UV-Vis absorption spectra were recorded in transmission mode. In these measurements, the gold NPs were dispersed in liquid solutions $(1 \mathrm{mg} / \mathrm{ml})$ : the $\mathrm{Au}-\mathrm{SC}_{12}$ and $\mathrm{Au}-\mathrm{SC}_{8}$ samples were dissolved in toluene, the $\mathrm{Au}-\mathrm{SC}_{8} / \mathrm{SC}_{11} \mathrm{COOH}$ sample was dissolved in ethanol and the $\mathrm{Au}-\mathrm{ST}$ nanoparticles were dissolved in water. The spectra were recorded in the range 200 to $850 \mathrm{~nm}$ with a Shimadzu UV-2102 PC spectrometer.

Magnetic measurements have been performed using a Quantum Design superconducting quantum interference device magnetometer. The sample holder is adhesive kapton which is sticked to a quartz tube. The diamagnetic contribution corresponding to the sample holder is previously measured and subtracted from the total magnetization.

\section{Results and discussion}

\subsection{Microstructural and chemical characterization}

The chemical composition of the samples is summarized in Table 1 as determined by ICP (Au and Fe content) and elemental chemical analysis (C, H, N and S content). The chemicals and solvents used in the NPs synthesis may contain iron impurities, typically, down to the ppm level; the synthesized samples may contain contamination from dispersed Fe which has its origin in the aforementioned impurities.

Table 1 Average size and chemical composition data for the selected samples.

\begin{tabular}{lccccccccc}
\hline Sample & $\begin{array}{c}\mathrm{Au} \\
\mathrm{wt} \%\end{array}$ & $\begin{array}{c}\mathrm{Fe} \\
\mathrm{wt} \%\end{array}$ & $\begin{array}{c}\mathrm{S} \\
\mathrm{wt} \%\end{array}$ & $\begin{array}{c}\mathrm{C} \\
\mathrm{wt} \%\end{array}$ & $\begin{array}{c}\mathrm{H} \\
\mathrm{wt} \%\end{array}$ & $\begin{array}{c}\mathrm{Au}: \mathrm{S} \\
\text { (at. ratio) }\end{array}$ & $\begin{array}{c}\mathrm{Dm}^{a} \\
(\mathrm{~nm})\end{array}$ & $\mathrm{Nr}_{\text {total }} b$ & $\mathrm{Nr}_{\text {surface }}{ }^{c}$ \\
\hline $\mathrm{Au}_{-} \mathrm{SC}_{12}$ & 50.3 & 0.007 & 4.1 & 24.3 & 4.4 & 2.0 & 2.0 & 177 & 69 \\
$\mathrm{Au}_{-} \mathrm{SC}_{8}$ & 86.3 & 0.096 & 4.5 & 13.9 & 2.3 & 3.1 & 2.3 & 249 & 65 \\
$\mathrm{Au}-\mathrm{ST}^{d}$ & 62.4 & 0.024 & 4.9 & 10.0 & 1.3 & 2.0 & 2.5 & 369 & 55 \\
$\mathrm{Au}-\mathrm{SC}_{8} / \mathrm{SC}_{11} \mathrm{COOH}$ & 52.4 & 0.180 & 6.2 & 29.3 & 4.3 & 1.5 & 1.9 & 141 & 70 \\
\hline
\end{tabular}

${ }^{a}$ Average particle size as calculated from particle size distribution histograms.

${ }^{b}$ Number of atoms calculated for a spherical pure gold cluster.

${ }^{c}$ Percentage of surface atoms calculated for a pure gold cluster.

${ }^{d}$ This sample contains some nitrogen from the amino group of the tiopronin molecule: $\mathrm{N}(\mathrm{wt} \%)=2.2$.

Fig. 1 contains a representative TEM micrograph from the Au-ST sample. These NPs have an average diameter of $2.5 \mathrm{~nm}$ as evaluated from the histogram; however, it has been 


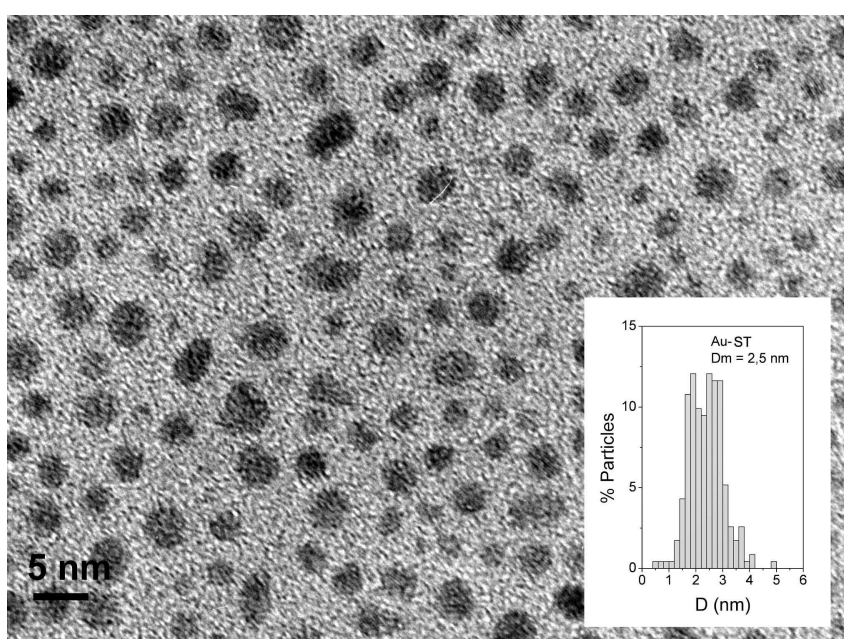

Fig. 1 TEM micrograph and particle size distribution histogram of the Au-ST sample.

found that very long exposures of these NPs to the electron beam can produce a slight increase of their size. The TEM micrographs of the $\mathrm{Au}-\mathrm{SC}_{12}$ and $\mathrm{Au}-\mathrm{SC}_{8}$ samples are shown in Fig. 2: the Au-SC $\mathrm{S}_{12}$ sample, already studied in a previous investigation by Guerrero et al (2007) that evaluated the NPs size effects in the magnetism, is here included to check how the length of the thiolated chain affects the physical properties. The average cluster diameter measured for these NPs is 2.0 and $2.3 \mathrm{~nm}$ respectively. The TEM micrograph of the $\mathrm{Au}-\mathrm{SC}_{8} / \mathrm{SC}_{11} \mathrm{COOH}$ sample is presented in Fig. 3, in this case, the average NP diameter determined from the histogram is $1.9 \mathrm{~nm}$.

Despite the $\mathrm{Au}-\mathrm{SC}_{8}$ sample is used as a seed in order to synthesize the $\mathrm{Au}-\mathrm{SC}_{8} / \mathrm{SC}_{11} \mathrm{COOH}$ sample, after the ligand exchange reaction, there is a noticeable decrease in the NP average diameter. Indeed, this size difference has been previously reported by Schaaff and Whetten (1999); the seed-NPs are dissolved in a solution that contains a new ligand, then an etching process starts in which gold atoms from the outermost surface layer of the seed-NP are removed from the metallic cluster core. The new NPs are smaller than the seed and the detached gold atoms can be found in a polymeric-like phase bonded to thiol ligands; this phase and the polymeric precursor which results from stopping the NP synthesis process just before a reducing agent $\left(\mathrm{NaBH}_{4}\right)$ is added are alike. In fact, Fig. 3 shows a more diffuse contrast around the NPs along with a blurred image of the metallic NPs core due to the existence of a polymeric-like phase.

\subsection{Electronic structure and microstructure}

The electronic structure, as well as the magnetic properties, of gold NPs are strongly dependent on their microstructure. Therefore, a XAS study of the samples has been carried out focusing on the analysis of the X-ray absorption near edge structure (XANES) data and the EXAFS region of the spectrum. Fig. 4 shows the normalized $A u L_{2}$-edge XANES of the four samples compared to both a bulk gold foil and a gold sulphide $\left(\mathrm{Au}_{2} \mathrm{~S}\right)$ reference samples. In this figure, the first resonance at the edge (around $5 \mathrm{eV}$ ) is the most significant feature of the XANES spectra. This resonance, also called white line, is related to a $2 p_{3 / 2,1 / 2} \rightarrow 5 d_{5 / 2,3 / 2}$ 

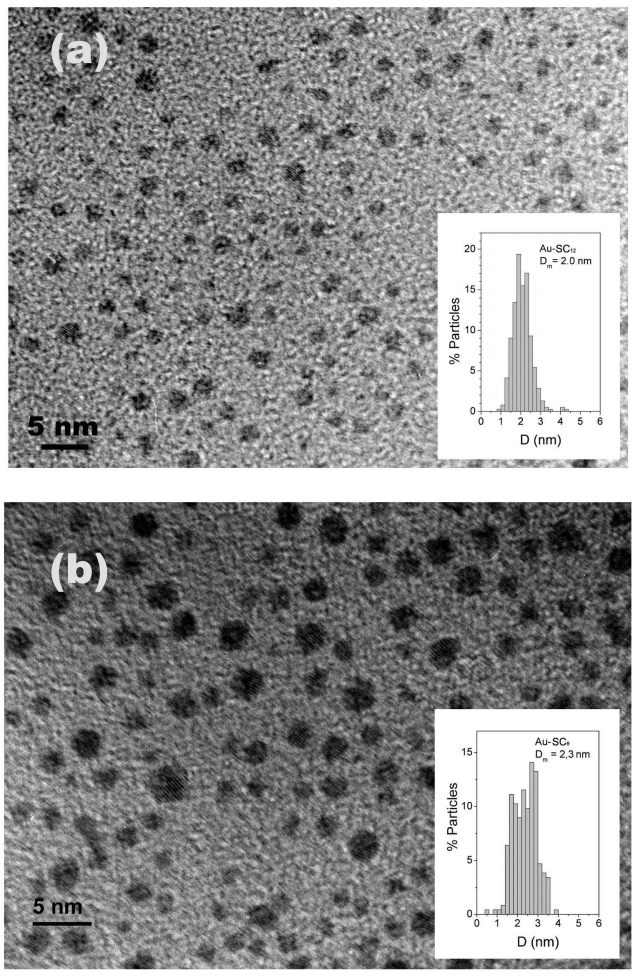

Fig. 2 TEM micrograph and particle size distribution histogram of the $\mathrm{Au}-\mathrm{SC}_{12}$ (a) and $\mathrm{Au}-\mathrm{SC}_{8}$ (b) samples.

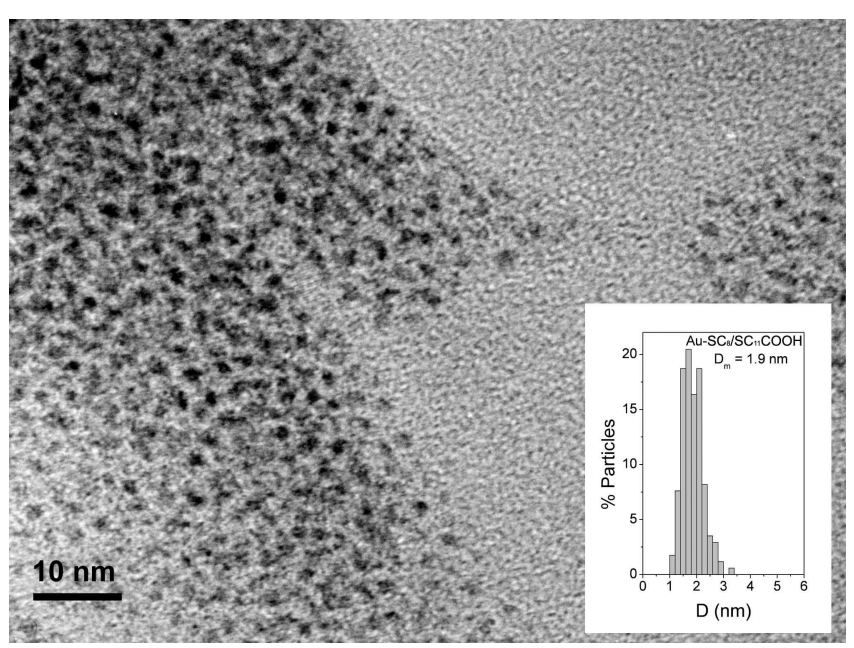

Fig. 3 TEM micrograph and particle size distribution histogram of the $\mathrm{Au}-\mathrm{SC}_{8} / \mathrm{SC}_{11} \mathrm{COOH}$ sample. 
dipole transition. Typically, the white line intensity is very strong for transition metals with a partially filled $d$-band and it gives an idea of the density of unoccupied states. However, the $5 d$ orbital of Au atoms is nominally full which means that the transition probability from a given energy level to a $5 d$ orbital is close to zero. Nevertheless, due to a $s-p-d$ hybridation, a faint white line can still be detected in the bulk gold XANES spectrum. A more intense white line relative to the bulk gold one indicates an increase of the $d$-hole population, i.e. charge depletion in the NPs just above the Fermi level (López-Cartes et al, 2005), as it happens in the studied systems. Regarding the meaning of this change in the $d$-hole population, it has been previously stated by Benfield et al (2001) that a change in the local geometry of a given system could lead to a variation on the hybridation of the valence orbitals which would be reflected as an intensity change of the white line. However, the systems studied in the current research work present XANES oscillations at the very same points as bulk gold do. This means that the investigated NPs have a crystalline fcc structure similar to bulk gold (c.f. Menard et al, 2006). Then, it could be said that any change in the white line intensity should be due to a charge transfer phenomenon between gold and sulphur, as Zhang and Sham (2003) have previously reported . Of course, this argument is valid only for clusters with fcc-like structure and, for instance, it could not be applied for very small NPs where the geometrical factor would produce a significant change in the valence orbitals hybridization and, subsequently, in the white line intensity. Such a modification in the atomic structure of nanometric particles, where different surface faceting effects appear depending on the nanoparticle size, has been already reported by Yacamán et al (2001). The latter assumption is used to justify the use of a gold sulphide reference sample to compare with the $\mathrm{Au}-\mathrm{SC}_{8} / \mathrm{SC}_{11} \mathrm{COOH}$ sample; both systems show similar XANES oscillations, therefore, they could be enclosed in the same structural group. In summary, the white line from bulk gold and $\mathrm{Au}_{2} \mathrm{~S}$ can be used to delimit the metallic and non-metallic character of the studied samples.

The $\mathrm{Au}-\mathrm{SC}_{8} / \mathrm{SC}_{11} \mathrm{COOH}$ sample presents a white line similar to the one from the gold sulphide reference (Fig. 4a), which suggests that this sample has a polymeric-like electronic structure; this is the electronic structure that would correspond to a polymeric precursor, as it has been previously described in previous publications (Menard et al, 2006; Guerrero et al, 2007). The gold found in the polymeric precursor happens to have an electronic behaviour far-off from the one of bulk gold, instead, the electronic properties of the polymeric precursor and the commercial gold sulphide are alike: the large number of gold atoms bonded to sulphur leads to a maximum value of the $d$-hole density in the Au atom. On the contrary, the samples labeled as $\mathrm{Au}-\mathrm{ST}, \mathrm{Au}-\mathrm{SC}_{12}$ and $\mathrm{Au}-\mathrm{SC}_{8}$ have almost the same faint white line which is comparable to the one from bulk gold (Fig. 4b). Due to the modification, through $\mathrm{Au}-\mathrm{S}$ bonds, of the outermost gold atoms in the NPs, their white line is always slightly more intense than the one from bulk gold.

In Fig. 5 the Fourier transforms of the EXAFS oscillations at the $\mathrm{Au} \mathrm{L}_{3}$-edge ( $k$ weighted, $k$ space range of 2.8-13.2 $\AA^{-1}$ without phase corrections) are shown. The spectra correspond to the $\mathrm{Au}-\mathrm{SC}_{12}$ and $\mathrm{Au}-\mathrm{SC}_{8}$ samples compared to the bulk gold (top) and to the Au-ST and $\mathrm{Au}-\mathrm{SC}_{8} / \mathrm{SC}_{11} \mathrm{COOH}$ samples compared to the gold sulphide reference sample (bottom). The spectrum of the Au-SC $\mathrm{SCC}_{8} \mathrm{COOH}$ sample shows a peak in the first coordination shell, around $2 \AA$, which corresponds to the Au-S bond, this peak is the most important contribution in this sample and confirms the existence of a -Au-S-Au-S- bond network. In addition, the $\mathrm{Au}-\mathrm{Au}$ coordination peaks, around 2.5 and $3.0 \AA$, can also be observed; regarding these peaks, the $\mathrm{Au}-\mathrm{ST}$ sample has stronger $\mathrm{Au}-\mathrm{Au}$ features than the $\mathrm{Au}-\mathrm{SC}_{8} / \mathrm{SC}_{11} \mathrm{COOH}$ sample. In principle, this result match the conclusions that can be extracted from the TEM micrographs where the Au-ST sample shows larger and better defined particles and the XANES 


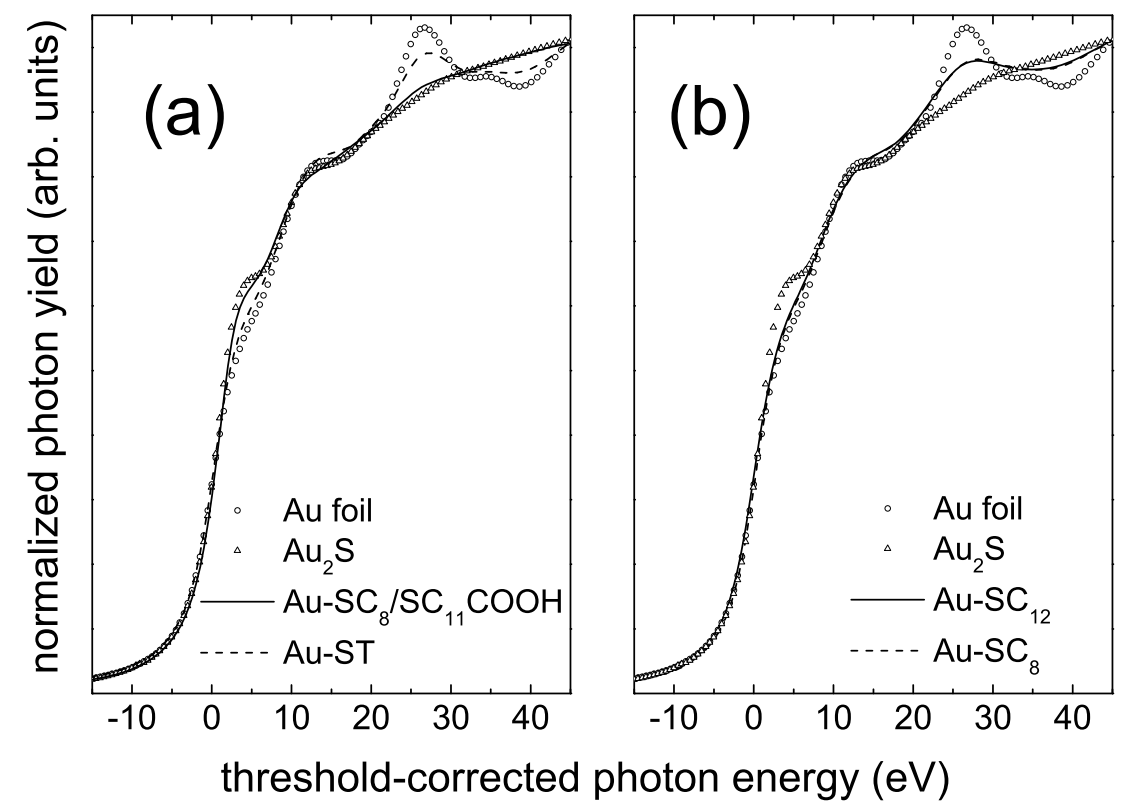

Fig. $4 \mathrm{Au} \mathrm{L}_{2}$-XANES spectra from the various samples compared to bulk gold (Au foil) and $\mathrm{Au}_{2} \mathrm{~S}$ reference samples: (a) the $\mathrm{Au}-\mathrm{SC}_{8} / \mathrm{SC}_{11} \mathrm{COOH}$ and $\mathrm{Au}-\mathrm{ST}$ samples; (b) the $\mathrm{Au}-\mathrm{SC}_{12}$ and $\mathrm{Au}-\mathrm{SC}_{8}$ samples.

spectra where, according to the white line intensity, the $\mathrm{Au}-\mathrm{SC}_{8} / \mathrm{SC}_{11} \mathrm{COOH}$ has a more

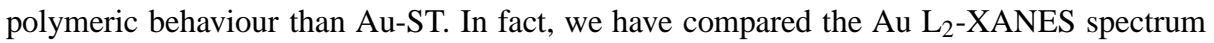
of the $\mathrm{Au}-\mathrm{SC}_{8} / \mathrm{SC}_{11} \mathrm{COOH}$ sample with a linear combination of the $\mathrm{Au} \mathrm{L}_{2}$-XANES spectra from the $\mathrm{Au}-\mathrm{SC}_{8}$ sample and a pure Au-SR polymeric material isolated during the synthesis of Au-SR nanoparticles. The results of such study conclude that a good agreement is achieved if a strong component (around 50\%) of polymer-like phase is present in the $\mathrm{Au}-$ $\mathrm{SC}_{8} / \mathrm{SC}_{11} \mathrm{COOH}$ sample.

For the $\mathrm{Au}-\mathrm{SC}_{12}$ and the $\mathrm{Au}-\mathrm{SC}_{8}$ samples, both the $\mathrm{Au}-\mathrm{S}$ coordination shell and the typical $\mathrm{Au}$-Au coordination peaks appear in the upper side of Fig. 5. The $\mathrm{Au}-\mathrm{SC}_{12}$ has a stronger $\mathrm{Au}-\mathrm{S}$ component; this is expected since the $\mathrm{Au}-\mathrm{SC}_{12}$ NPs are slightly smaller than the $\mathrm{Au}-$ $\mathrm{SC}_{8}$. Therefore, the number of gold surface atoms bonded to sulphur is proportionally larger compared to the number of gold atoms bonded to gold atoms. The Au-ST sample shows the smallest Au-S component as corresponds to a NP with the largest average diameter.

The quantification of the EXAFS observations is presented in Fig. 6 where experimental data and simulation results of the EXAFS oscillations and radial distribution functions are included. In this figure, every investigated sample is presented but the reference samples: bulk gold and gold sulphide. The results of the EXAFS simulations are summarized in Table 2; the following structural parameters were determined: coordination number $(\mathrm{N})$, bond distance $(\mathrm{R})$ and Debye-Waller factor $(\sigma)$. The $\mathrm{Au}-\mathrm{Au}$ and $\mathrm{Au}-\mathrm{S}$ coordination numbers of the $\mathrm{Au}-\mathrm{SC}_{8}$ and $\mathrm{Au}-\mathrm{SC}_{12}$ samples are consistent with the particle size decrease along with an increase in the number of gold atoms bonded to sulphur. In addition, the Au-Au bond length 


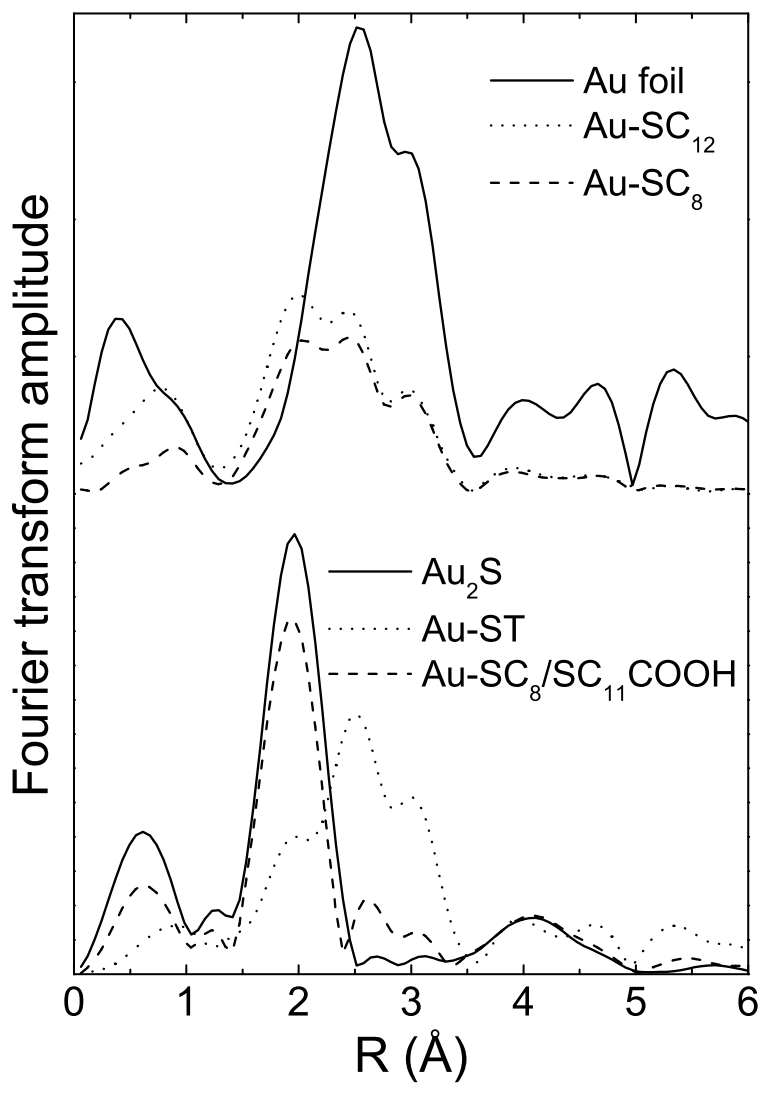

Fig. 5 Modulus of the Fourier Transform of EXAFS oscillations at the Au- $\mathrm{L}_{3}$-edge ( $k$ weighted, $k$ space range of 2.8-13.2 $\AA^{-1}$ without phase corrections) for the $\mathrm{Au}_{-} \mathrm{SC}_{12}, \mathrm{Au}-\mathrm{SC}_{8}, \mathrm{Au}-\mathrm{ST}$ and $\mathrm{Au}-\mathrm{SC}_{8} / \mathrm{SC}_{11} \mathrm{COOH}$ samples compared to bulk gold (Au foil) and $\mathrm{Au}_{2} \mathrm{~S}$ reference samples.

has been estimated for these samples and it is $2.77 \AA$, which is shorter than the evaluated distance for bulk gold $(2.85 \AA)$. This bond length difference indicates a lattice contraction already reported by Zhang and Sham (2002) for small gold clusters. Regarding the Au-ST sample which contains larger particles, the Au-Au coordination number increases and the Au-Au bond distance, being $2.80 \AA$, is closer to the bulk value.

Very precise microstructural information has been obtained from the transmission electron microscopy and the XAS data. This has been very important when interpreting the UV-Vis absorption behaviour of the samples. Typically, surface plasmon resonance bands (hereafter SPR) dominate the 400-800 $\mathrm{nm}$ wavelength range of gold nanoparticles UV-Vis absorption spectrum. As the particles become smaller, a higher proportion of surface gold atoms bonded to sulphur lead to charge localization at the $\mathrm{Au}-\mathrm{S}$ bond that decreases the mobility of free electrons (García et al, 2005) and the absorption intensity at the plasmon region. In fact, the UV-Vis spectrum of the larger alkanethiolated nanoparticle $\left(\mathrm{Au}-\mathrm{SC}_{8}\right)$ shows a weak plasmon resonance around $520 \mathrm{~nm}$ (c.f. Fig. 7), whereas the absorbance of the smaller 
Table 2 Best fitting parameters of the $\mathrm{Au} \mathrm{L}_{3}$-edge EXAFS oscillations of samples.

\begin{tabular}{lccccc}
\hline Sample & Elements $^{a}$ & $\mathrm{~N}^{b}$ & $\begin{array}{c}\mathrm{R}(\AA)^{c} \\
( \pm 0.02 \AA)\end{array}$ & $\sigma(\AA)^{d}$ & $\begin{array}{c}\Delta \sigma^{2} \times 10^{-3} \\
\left(\AA^{2}\right)\end{array}$ \\
\hline Au foil & $(\mathrm{Au}-\mathrm{Au})_{m}$ & 12 & 2.85 & $0.073 \pm 0.015$ & \\
$\mathrm{Au}_{2} \mathrm{~S}$ & $(\mathrm{Au}-\mathrm{S})$ & $1.9 \pm 0.4$ & 2.31 & $0.063 \pm 0.013$ & \\
$\mathrm{Au}-\mathrm{SC}_{12}$ & $(\mathrm{Au}-\mathrm{S})$ & $0.75 \pm 0.15$ & 2.30 & $0.093 \pm 0.019$ & \\
& $(\mathrm{Au}-\mathrm{Au})_{m}$ & $5.0 \pm 1.0$ & 2.77 & $0.078 \pm 0.016$ & 0.8 \\
$\mathrm{Au}-\mathrm{SC}_{8}$ & $(\mathrm{Au}-\mathrm{S})$ & $0.58 \pm 0.12$ & 2.30 & $0.10 \pm 0.02$ & \\
& $(\mathrm{Au}-\mathrm{Au})_{m}$ & $5.60 \pm 1.12$ & 2.77 & $0.091 \pm 0.018$ & 3.0 \\
$\mathrm{Au}-\mathrm{ST}$ & $(\mathrm{Au}-\mathrm{S})$ & $0.48 \pm 0.10$ & 2.26 & $0.090 \pm 0.018$ & \\
& $(\mathrm{Au}-\mathrm{Au})_{m}$ & $8.9 \pm 1.8$ & 2.80 & $0.089 \pm 0.018$ & 2.6 \\
$\mathrm{Au}-\mathrm{SC}_{8} / \mathrm{SC}_{11} \mathrm{COOH}$ & $(\mathrm{Au}-\mathrm{S})$ & $1.0 \pm 0.2$ & 2.30 & $0.050 \pm 0.010$ & \\
& $(\mathrm{Au}-\mathrm{Au})_{m}$ & $2.0 \pm 0.4$ & 2.80 & $0.085 \pm 0.017$ & 1.9 \\
\hline
\end{tabular}

${ }^{a}$ Type of atoms in the coordination shell.

${ }^{b}$ Coordination number.

${ }^{c}$ Bond length.

${ }^{d}$ Debye-Waller factor.

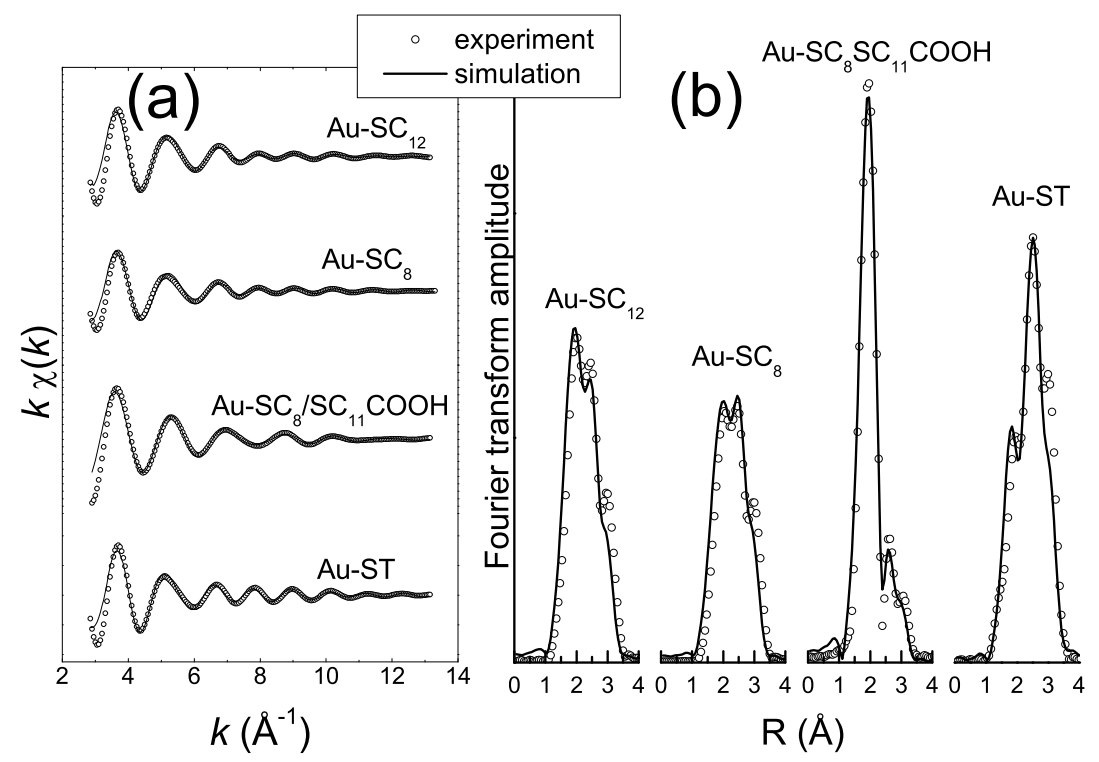

Fig. 6 Experimental and simulated EXAFS oscillations and radial distribution functions for $\mathrm{Au}-\mathrm{SC}_{12}, \mathrm{Au}^{-}$ $\mathrm{SC}_{8}, \mathrm{Au}-\mathrm{SC}_{8} / \mathrm{SC}_{11} \mathrm{COOH}$ and $\mathrm{Au}-\mathrm{ST}$ samples.

clusters decay in the visible region. The Au-ST sample, despite having the largest nanoparticles, does not show any plasmon resonance around $520 \mathrm{~nm}$. This result agrees with the UV-Vis spectra reported by Templeton et al (1999) where the SPR band intensity is lower for tiopronin-functionalized NPs than for alkanethiol-protected particles of similar size. This effect has been associated to a lack of order in the tiopronin capping system and may also be related to differences in the dielectric properties of alkanethiolated and tiopronin monolay- 


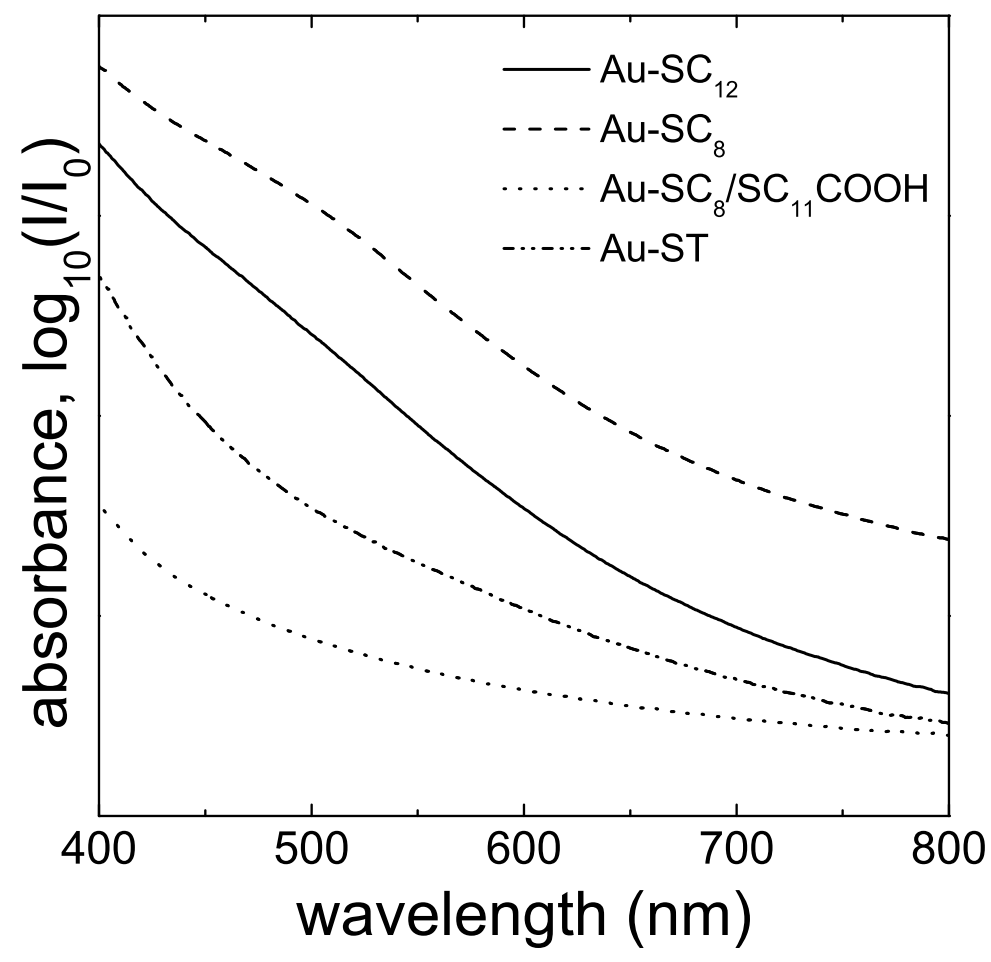

Fig. 7 UV-Visible absorption spectra for the $\mathrm{Au}-\mathrm{SC}_{12}, \mathrm{Au}-\mathrm{SC}_{8}, \mathrm{Au}-\mathrm{SC}_{8} / \mathrm{SC}_{11} \mathrm{COOH}$ and Au-ST samples.

ers; its origin is still unclear and needs further analysis. For the $\mathrm{Au}-\mathrm{SC}_{8} / \mathrm{SC}_{11} \mathrm{COOH}$ sample, the presence of a polymeric-like phase produces a strong decrease in UV-Vis absorption due to the lost of metallic character and to the total charge localization at the -Au-S-Au-S- bonds.

\subsection{Magnetic properties}

Magnetization curves measured at two different temperatures ( 5 and $300 \mathrm{~K}$ ) on the $\mathrm{Au}-\mathrm{SC}_{12}$, $\mathrm{Au}-\mathrm{SC}_{8}, \mathrm{Au}-\mathrm{ST}$ and $\mathrm{Au}-\mathrm{SC}_{8} / \mathrm{SC}_{11} \mathrm{COOH}$ samples are presented in Fig. 8. The physical magnitudes, estimated from the measured hysteresis cycles, are summarized in Tab. 3. The magnetization is normalized to the estimated mass of bonded gold which was determined from the ICP analysis results. Since the Au:S ratio is greater than one for every studied sample, it has been assumed that all $\mathrm{Au}$ atoms located at the nanoparticle surface are bonded to a thiol group. Even at room temperature, samples $\mathrm{Au}-\mathrm{SC}_{12}$ and $\mathrm{Au}-\mathrm{SC}_{8}$ clearly exhibit a ferromagnetic-like behaviour. The magnetization values at $5 \mathrm{~K}$ are quite similar for samples $\mathrm{Au}-\mathrm{SC}_{12}$ and $\mathrm{Au}-\mathrm{SC}_{8}, M_{s}=0.135 \mathrm{emu} / \mathrm{g} \mathrm{Au}$ bonded and $M_{s}=0.105 \mathrm{emu} / \mathrm{g} \mathrm{Au}_{\text {bonded }}$ respectively, which would be equivalent to say that magnetization is almost the same for 
Table 3 Magnetic properties for the selected samples estimated from hysteresis cycles measured at $T=5 \mathrm{~K}$.

\begin{tabular}{lccccc}
\hline Sample & $\begin{array}{c}\mathrm{Au}_{\text {bonded }}{ }^{a} \\
\mathrm{wt} \%\end{array}$ & $\begin{array}{c}M_{s}{ }^{b} \\
(\mathrm{emu} / \mathrm{g} \mathrm{Au} \text { bonded })\end{array}$ & $\begin{array}{c}\mu_{\mathrm{Au}}{ }^{c} \\
\left(\mu_{B}\right)\end{array}$ & $\begin{array}{c}\mathrm{V}_{\mathrm{Au}}{ }^{d} \\
\left(\mathrm{~nm}^{3}\right)\end{array}$ & $\begin{array}{c}K^{e} \\
\left(10^{7} \mathrm{~J} / \mathrm{m}^{3}\right)\end{array}$ \\
\hline $\mathrm{Au}^{3} \mathrm{SC}_{12}$ & 34.7 & 0.135 & 0.005 & 2.9 & 3.6 \\
$\mathrm{Au}-\mathrm{SC}_{8}$ & 56.0 & 0.105 & 0.004 & 4.1 & 2.5 \\
$\mathrm{Au}-\mathrm{ST}$ & 62.4 & 0.03 & 0.001 & 4.5 & paramagnetic \\
$\mathrm{Au}-\mathrm{SC}_{8} / \mathrm{SC}_{11} \mathrm{COOH}^{f}$ & 36.8 & 0.016 & 0.0006 & 2.6 & 4.0 \\
\hline
\end{tabular}

${ }^{a}$ Estimated amount of gold atoms bonded to sulphur atoms.

${ }^{b}$ Magnetic saturation.

${ }^{c}$ Magnetic moment per bonded gold atom, expressed in Bohr magneton units $\mu_{B}$.

${ }^{d}$ Estimated volume of gold atoms bonded to thiol groups.

${ }^{e}$ Anisotropy constant.

${ }^{f}$ Estimation of dispersed Au atoms in the polymeric phase is not possible, therefore it is assumed that all $\mathrm{Au}$ atoms in this sample are within the NPs core.

samples protected with a similar organic chain. Coercitive fields of 90 and 49 Oe were also measured for the $\mathrm{Au}-\mathrm{SC}_{12}$ and the $\mathrm{Au}-\mathrm{SC}_{8}$ samples respectively. From the previously determined values, the magnetic moment of a bonded Au atom is estimated to be $\mu_{A u}=0.005 \mu_{B}$ and $\mu_{A u}=0.004 \mu_{B}$, for the $\mathrm{Au}-\mathrm{SC}_{12}$ and $\mathrm{Au}-\mathrm{SC}_{8}$ samples respectively. The quite similar values of magnetic moment provide further evidence that the appearance of a net magnetic moment should be undoubtedly attributed to the charge transfer that occurs through the $\mathrm{Au}-$ $\mathrm{S}$ bond. Moreover, it also depends on the geometry/structure of the organic capping chains. It is worth to remark that magnetic hysteresis is observed at room temperature for samples $\mathrm{Au}-\mathrm{SC}_{12}, \mathrm{Au}-\mathrm{SC}_{8}$ and $\mathrm{Au}-\mathrm{SC}_{8} / \mathrm{SC}_{11} \mathrm{COOH}$ which implies that magnetic moments should be "frozen" due to an enormous local anisotropy field, this means that the anisotropy constant should be, at least, $10^{7} \mathrm{~J} / \mathrm{m}^{3}$. The anisotropy constant value is estimated by comparing thermal energy, $k_{B} T$ where $k_{B}$ is the Boltzmann constant and, anisotropy energy, $K V$ where $K$ stands for the anisotropy constant and $V$ for the volume occupied by surface Au atoms bonded to thiol groups. The estimated $K$ values are summarized in Tab. 3 and should be taken as a lower limit since it has been determined in order to show ferromagnetic-like behaviour even at $T=300 \mathrm{~K}$. Clearly, higher $K$ values are also feasible since they would also be compatible with having ferromagnetic-like properties at room temperature.

Despite charge transfer between gold and sulphur, which was determined by XANES measurements, and the high percentage of dispersed gold atoms bonded to sulphur in the $\mathrm{Au}-\mathrm{SC}_{8} / \mathrm{SC}_{11} \mathrm{COOH}$ sample, the magnetization $\left(M_{S}=0.016 \mathrm{emu} / \mathrm{g} \mathrm{Au}_{\text {bonded }}\right)$ is one order of magnitude smaller than the magnetization measured for alkanethiol-capped Au NPs. This peculiar behaviour should be linked with having a considerable amount of $\mathrm{Au}$ atoms in a polymeric-like phase, i.e. bonded to thiolated ligands. Recently, the magnetic behaviour of pure polymeric-like samples was studied, such research work showed that polymeric-like samples exhibited a very weak magnetic signal. From this work by Guerrero et al (2007), it was concluded that the simultaneous presence of Au-Au and Au-S bonds is essential in order to have a significant ferromagnetic-like behaviour.

Finally, for Au NPs stabilized with tiopronin, the magnetization curve is quite different from the previous curves. In this case no ferromagnetic behaviour is observed, instead, there is a linear dependence of the magnetization with the applied external field. The magnetization is $M_{s}=0.03 \mathrm{emu} / \mathrm{g} \mathrm{Au}$ bonded when a $1 \mathrm{~T}$ external magnetic field is applied. The absence of ferromagnetic-like behaviour indicates that no anisotropy field is present in these nanoparticles. 

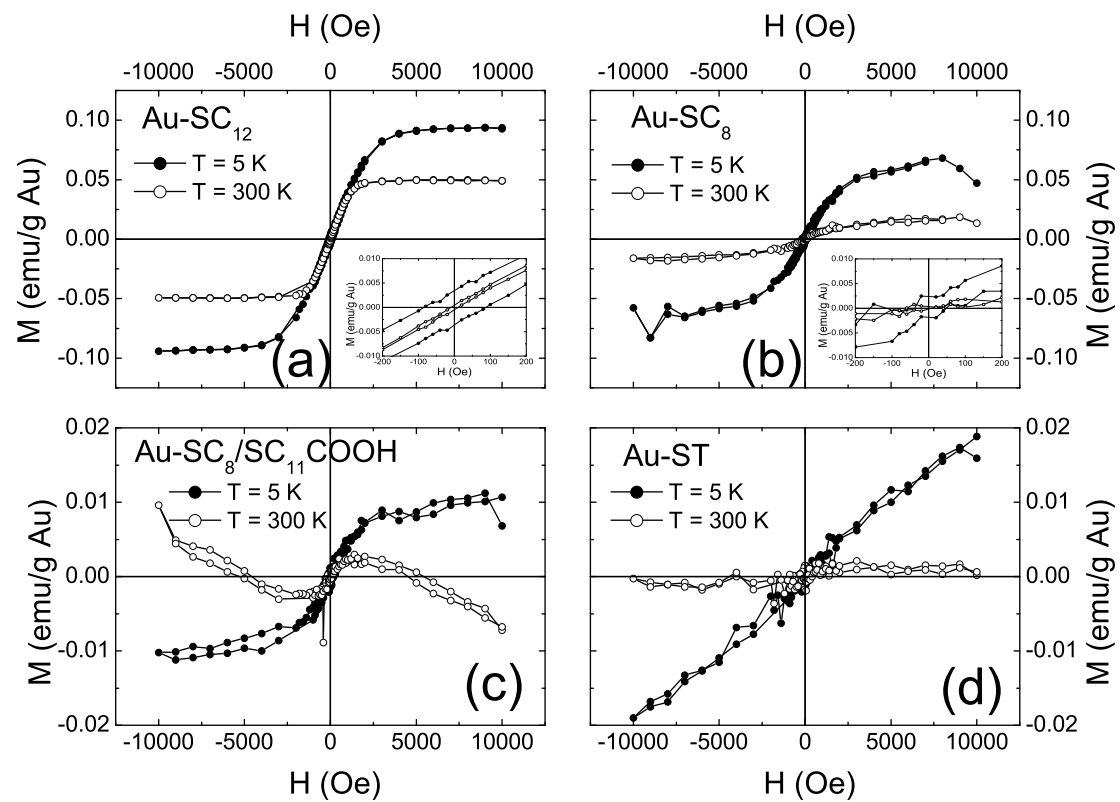

Fig. 8 Hysteresis loops corresponding to the gold thiol-capped nanoparticles $\mathrm{Au}-\mathrm{SC}_{12}$ (a), Au-SC 8 (b), Au$\mathrm{SC}_{8} / \mathrm{SC}_{11} \mathrm{COOH}(\mathrm{c})$ and $\mathrm{Au}-\mathrm{ST}(\mathrm{d})$. The measurements were performed at two different temperatures: 5 and $300 \mathrm{~K}$.

The magnetization curves summarized in Fig. 8 clearly indicate that although the charge transfer between gold and thiol groups is the responsible for the appearance of a nonnegligible magnetic moment, the anisotropy field responsible for the ferromagnetic-like behaviour is determined not only by the bond nature but also by the capping molecule. Since all Au NPs are capped with thiol-functionalized molecules, similar magnetic features should be expected for all the samples, such magnetic properties shall undergo slight variations arising from the different Au:S ratio, as well as particle diameters. In previous studies, it has been demonstrated that magnetism appears when organic molecules form self-assembled monolayers on gold substrates. Therefore, since the white line intensity of the Au NPs stabilized with tiopronin is very similar to the measured intensity for alkanethiol-capped Au NPs, the absence of ferromagnetism in Au NPs capped with tiopronin should be related with the lack of order in the chemisorbed layer as reported by Templeton et al (1999).

Samples capped with alkanethiol molecules exhibit the highest values of magnetization. While NPs from the Au-SC 12 sample are bonded to twelve-carbon chains, NPs from the $\mathrm{Au}-\mathrm{SC}_{8}$ sample are bonded to eight-carbon chains; since both NPs have a very similar diameter, this experiment would be a test to check how the thiolated chain length affects the magnetic behaviour. The results are slightly different for both samples, in fact, the $\mathrm{Au}-\mathrm{SC}_{12}$ sample shows a higher magnetization, as well as a higher coercitive field, than the $\mathrm{Au}-\mathrm{SC}_{8}$ sample. The effect can be related to a slight increase in the charge transfer value from $\mathrm{Au}$ to $\mathrm{S}$ when increasing the length of the alkanethiol chain and also to differences in the degree of order during the formation of the self-assembled protective shell. It is also worth 
mentioning that for these two samples a broad surface plasmon resonance band is detected, while for the Au-ST sample, even if it is the nanoparticle with the largest diameter, such a feature is not present. Thus, as the SPR band in the absorption spectrum indicates, the absence of anisotropy field in the Au-ST sample could be related with the lack of order in the capping system, in contrast with the formation of alkanethiol self-assembled monolayers on gold surfaces. Concerning the $\mathrm{Au}-\mathrm{SC}_{8} / \mathrm{SC}_{11} \mathrm{COOH}$ sample, which is obtained from the $\mathrm{Au}-\mathrm{SC}_{8}$ sample, some differences should arise from the formation of the polymeric phase which favours the absence of the SPR feature. Besides, the white line intensity of this sample has the highest value of them all and, in addition, the magnetization values are one order of magnitude lower than the rest. This behaviour is attributed to the presence of the polymeric phase in which $\mathrm{Au}$ atoms are bonded to $\mathrm{S}$ atoms, but no $\mathrm{Au}-\mathrm{Au}$ bonds are formed. Hence, this result again indicates that the ferromagnetic-like behaviour is connected to particle formation, i.e. to the existence of a metallic nanoparticle. This supports the model proposed by Hernando et al (2006a,b) which accounts for such a high anisotropy field. This model takes into account the high values of the spin-orbit interaction at gold surfaces (LaShell et al, 1996) and the fact that, according to the SPR band, in the samples that exhibit ferromagnetic behaviour the Au-S bond generates $5 d$ localized holes. It is proposed that the localized charge or spin that is originated due to the Au-S bond at the nanoparticle surface, drives the induction of orbital momentum at the conduction electrons to minimize spin-orbit interaction. The giant anisotropy to which the localized spins are exposed to is due to a strong effective magnetic field which has its origin on the coupling to $l_{z}$ via spin-orbit coupling.

The ICP analysis shows that there is Fe contamination in our samples always. However, the level of $\mathrm{Fe}$ impurities cannot explain the observed magnetization behaviour since the sample with the lowest $\mathrm{Fe}$ content $\left(\mathrm{Au}-\mathrm{SC}_{12}\right)$ has the strongest ferromagnetic feature. In addition, recent experiments by Crespo et al (2006) have reported that magnetic impurities, far from inducing a ferromagnetic behaviour, reduce the ferromagnetic order temperature in thiol-capped $\mathrm{Au}$ glyconanoparticles. Also in the case of the $\mathrm{Au}-\mathrm{SC}_{12}$ sample, the total amount of found iron could not account for the measured magnetization even if we consider only Fe concentrated in metallic particles, which is likely not the case.

\section{Conclusions}

Gold NPs have been stabilized with a variety of thiol functionalized molecules in order to modify their chemical and physical properties. Following the Brust method, alkanethiolatecapped NPs have been obtained with two different thiolate chain lengths. Water-soluble tiopronin-capped NPs were obtained following the method proposed by Templeton. The NPs capped with the shortest alkanethiol chain were modified in order to include also thiolated chains bonded to a carboxylic group. This has shown that besides the magnetic properties of the synthesized gold NPs, due to their thiol-protecting shells with different functional groups and solubility properties in organic and aqueous solvents, these NPs have a clear potential in many applications.

At the same time, we have carried out a thorough study of the microstructure, chemical composition and magnetic properties that has allowed us to determine some key factors responsible of the singular ferromagnetic behaviour found in thiol functionalized gold NPs. In agreement with a previous investigation (Guerrero et al, 2007), it has been experimentally proven that the simultaneous presence of $\mathrm{Au}-\mathrm{Au}$ and $\mathrm{Au}-\mathrm{S}$ bonds is necessary to detect ferromagnetic behaviour in thiol stabilized nanostructures. Polymeric-like phases (-Au-S$\mathrm{Au}-\mathrm{S}$ - bonds) do not show magnetization features. In addition, the formation of ordered 
self-assembled monolayer domains at the protective shell is another fundamental parameter necessary to observe a ferromagnetic-like behaviour. These results would support the theory proposed by Hernando et al (2006a) which explains the arising of magnetization via induced orbital momentum.

Finally, for alkanethiol functionalized NPs, we also found a variation of the magnetic properties with the thiol chain length. The differences are small, however, a slight tendency was observed for a decrease in magnetization when shortening the alkane chain.

Acknowledgements We wish to acknowledge the support of the ESRF and BM29 beamline staff. Financial support from the Spanish MEC (NAN2004-09125-C07) and "Junta de Andalucía” (Project P06-FQM-02254, group TEP217) is also acknowledged. E. Guerrero thanks the Spanish MEC for financial support.

\section{References}

Benfield RE, Grandjean D, Kröll M, Pugin R, Sawitowski T, Schmid G (2001) Structure and bonding of gold metal clusters, colloids, and nanowires studied by EXAFS, XANES and WAXS. J Phys Chem B 105:1961-1970

Bonin D, Kaiser P, Freitigny C, Desbarres J (1989) Logiciels d'analyse EXAFS. In: Dexpert $\mathrm{H}$, Michalowizc A, Verdagner M (eds) Structures fines d'absorption des rayons $\mathrm{X}$ en chimie, 3, Societé Française de Chimie, Paris

Brust M, Walker M, Bethell D, Schiffrin DJ, Whyman R (1994) Synthesis of thiolderivatised gold nanoparticles in a two-phase liquid-liquid system. J Chem Soc, Chem Commun 7:801-802

Carmeli I, Leitus G, Naaman R, Reich S, Vager Z (2003) Magnetism induced by the organization of self-assembled monolayers. J Chem Phys 118:10,372-10,375

Chushak Y, Bartell L (2000) Molecular dynamics simulations of the freezing of gold nanoparticles. Eur Phys J D 16:43-46

Crespo P, Litrán R, Rojas TC, Multigner M, de la Fuente JM, Sánchez-López JC, García MA, Hernando A, Penadés S, Fernández A (2004) Permanent magnetism, magnetic anisotropy, and hysteresis of thiol-capped gold nanoparticles. Phys Rev Lett 93:087,204(4)

Crespo P, García MA, Fernández-Pinel E, Multigner M, Alcántara D, de la Fuente JM, Penadés S, Hernando A (2006) Fe impurities weaken the ferromagnetic behavior in Au nanoparticles. Phys Rev Lett 97:177,203(4)

de la Fuente JM, Berry CC, Riehle MO, Curtis ASG (2006) Nanoparticle targeting at cells. Langmuir 22:3286-3293

García MA, de la Venta J, Crespo P, Llopis JJ, Penadés S, Fernández A, Hernando A (2005) Surface plasmon resonance of capped Au nanoparticles. Phys Rev B 72:R241,403(4)

Guerrero E, Rojas TC, Multigner M, Crespo P, Muñoz-Márquez MA, García MA, Hernando A, Fernández A (2007) Evolution of the microstructure, chemical composition and magnetic behaviour during the synthesis of alkanethiol-capped gold nanoparticles. Acta Mat 55:1723-1730

Hernando A, García MA (2006) Comment on 'Bosons as the origin for giant magnetic properties of organic monolayers'. Phys Rev Lett 96:029,703(1)

Hernando A, Crespo P, García MA (2006a) Origin of orbital ferromagnetism and giant magnetic anisotropy at the nanoscale. Phys Rev Lett 96:057,206(4) 
Hernando A, Crespo P, García MA, Fernández-Pinel E, de la Venta J, Fernández A, Penadés S (2006b) Giant magnetic anisotropy at the nanoscale: overcoming the superparamagnetic limit. Phys Rev B 74:052,403(4)

Hernando A, Sampedro B, Litrán R, Rojas TC, Sánchez-López JC, Fernández A (2006c) Room temperature permanent magnetism in thiol-capped Pd-rich nanoparticles. Nanotechnology 17:1449-1453

Hori H, Teranishi T, Nakae Y, Seino Y, Miyake M, Yamada S (1999) Anomalous magnetic polarization effect of Pd and Au nano-particles. Phys Lett A 263:406-410

Iwasa T, Nobusada K (2007) Gold-thiolate core-in-cage cluster $\mathrm{Au}_{25}\left(\mathrm{SCH}_{3}\right)_{18}$ shows localized spins in charged states. Chem Phys Lett 441:268-272

Kondo Y, Takayanagi K (1997) Gold nanobridge stabilized by surface structure. Phys Rev Lett 79:3455-3458

Kondo Y, Ru Q, Takayanagi K (1999) Thickness induced structural phase transition of gold nanofilm. Phys Rev Lett 82:751-754

Koskinen P, Häkkinen H, Seifert G, Sanna S, Frauenheim T, Moseler M (2006) Densityfunctional based tight-binding study of small gold clusters. New J Phys 8:1-11

Kumar V, Kawazoe Y (2003) Magnetism in clusters of non-magnetic elements: Pd, Rh, and Ru. Eur Phys J D 24:81-84

LaShell S, McDougall BA, Jensen E (1996) Spin splitting of an Au(111) surface state band observed with angle resolved photoelectron spectroscopy. Phys Rev Lett 77:3419-3422

Litrán R, Sampedro B, Rojas TC, Multigner M, Sánchez-López JC, Crespo P, López-Cartes C, García MA, Hernando A, Fernández A (2006) Magnetic and microstructural analysis of palladium nanoparticles with different capping systems. Phys Rev B 73:054,404(7)

López-Cartes C, Rojas TC, Litrán R, Martínez-Martínez D, de la Fuente JM, Penadés S, Fernández A (2005) Gold nanoparticles with different capping systems: an electronic and structural XAS analysis. J Phys Chem B 109:8761-8766

Menard LD, Xu H, Gao SP, Twesten RD, Harper AS, Song Y, Wang G, Douglas AD, Yang JC, Frenkel AI, Murray RW, Nuzzo RG (2006) Metal core bonding motifs of monodisperse icosahedral $\mathrm{Au}_{13}$ and larger $\mathrm{Au}$ monolayer-protected clusters as revealed by X-ray absorption spectroscopy and transmission electron microscopy. J Phys Chem B 110:14,564-14,573

Mitome M, Takayanagi K, Tanishiro Y (1990) Commensurate reconstruction on a si(001) facet of a gold particle. Phys Rev B 42:7238-7241

Negishi Y, Tsunoyama H, Suzuki M, Kawamura N, Matsushita MM, Maruyama K, Sugawara T, Yokoyama T, Tsukuda T (2006) X-ray magnetic circular dichroism of sizeselected, thiolated gold clusters. J Am Chem Soc 128:12,034-12,035

Petersen L, Hedegard P (2000) A simple tight-binding model of spin-orbit splitting of $s p$ derived surface states. Surf Sci 459:49-56

Reich S, Leitus G, Feldman Y (2006) Observation of magnetism in Au thin films. Appl Phys Lett 88:222,502(3)

Sampedro B, Crespo P, Hernando A, Litrán R, Sánchez-López JC, López-Cartes C, Fernández A, Ramírez J, González-Calbet J, Vallet M (2003) Ferromagnetism in fcc twinned $2.4 \mathrm{~nm}$ size Pd nanoparticles. Phys Rev Lett 91:237,203(4)

Schaaff TG, Whetten RL (1999) Controlled etching of Au:SR cluster compounds. J Phys Chem B 103:9394-9396

Shinohara T, Sato T, Taniyama T (2003) Surface ferromagnetism of Pd fine particles. Phys Rev Lett 91:197,201(4)

Simard J, Briggs C, Boal AK, Rotello VM (2000) Formation and pH-controlled assembly of amphiphilic gold nanoparticles. Chem Comm 19:1943-1944 
Templeton AC, Chen S, Gross SM, Murray RW (1999) Water-soluble, isolable gold clusters protected by tiopronin and coenzyme A monolayers. Langmuir 15:66-76

Vager Z, Naaman R (2004) Bosons as the origin for giant magnetic properties of organic monolayers. Phys Rev Lett 92:087,205(4)

Yacamán MJ, Ascencio JA, Liu H, Gardea-Torresdey J (2001) Structure shape and stability of nanometric sized particles. J Vac Sci Technol B 19:1091-1103

Yamamoto Y, Miura T, Suzuki M, Kawamura N, Miyagawa H, Nakamura T, Kobayashi K, Teranishi T, Hori H (2004) Direct observation of ferromagnetic spin polarization in gold nanoparticles. Phys Rev Lett 93:116,801(4)

Yu M, Satterley CJ, Bengió S, Lovelock KRJ, Milligan PK, Jones RG, Woodruff DP, Dhanak V (2006) True nature of an archetypal self-assembly system: mobile au-thiolate species on au(111). Phys Rev Lett 97:166,102(4)

Zabinsky SI, Rehr JJ, Ankudinov A, Albers RC, Eller MJ (1995) Multiple-scattering calculations of x-ray-absorption spectra. Phys Rev B 52:2995-3009

Zhang P, Sham TK (2002) Tuning the electronic behavior of Au nanoparticles with capping molecules. Appl Phys Lett 81:736-738

Zhang P, Sham TK (2003) X-ray studies of the structure and electronic behavior of alkanethiolate-capped gold nanoparticles: the interplay of size and surface effects. Phys Rev Lett 90:245,502(4) 